\title{
The Inclusion of Spillover Effects in Economic Evaluations: Not an Optional Extra
}

\author{
Werner B. F. Brouwer ${ }^{1,2,3}$ \\ (c) Springer Nature Switzerland AG 2018
}

\section{Introduction}

Increasingly, welfare economic evaluations are used in the context of the allocation of scarce healthcare resources. Many jurisdictions prescribe their use in the context of reimbursement, funding, and/or pricing of new health technologies, especially pharmaceuticals. The fact that the outcomes of such evaluations can influence actual allocation decisions in healthcare underlines the importance of a sound methodology and an appropriate decision-making process. Fortunately, in both areas progress has been made over the past decades. However, there remains room for further improvement, for instance in finding appropriate estimates of monetary values of health, developing broader outcome measures than quality-adjusted life-years (QALYs), finding appropriate equity (principles to estimate) weights for health outcomes, and estimating health opportunity costs.

A topic that receives increasing attention in this context is the inclusion in economic evaluations of so-called spillover effects [1-5]. These include the health and wellbeing effects of illness, and treatment of patients on 'significant others', most importantly family members and informal caregivers [4]. These effects are commonly ignored in economic evaluations, which typically treat patients as isolated individuals, ignoring potentially large wellbeing and health impacts their illness can have on family members and caregivers [2,

This Commentary has a reply available at doi: https://doi. org/10.1007/s40273-018-0729-z.

Werner B. F. Brouwer

brouwer@eshpm.eur.nl

1 Erasmus School of Health Policy \& Management, Erasmus University Rotterdam, PO Box 1738, 3000 DR Rotterdam, The Netherlands

2 Erasmus School of Economics, Erasmus University Rotterdam, Rotterdam, The Netherlands

3 Institute for Medical Technology Assessment, Erasmus University Rotterdam, Rotterdam, The Netherlands
$3,6,7]$. In this commentary, I argue that it is necessary to include these effects in economic evaluations for reasons of efficiency and equity. This is true for both economic evaluations taking a societal perspective and those taking a narrower healthcare perspective. Further investigation of their measurement, valuation, and consideration in the decisionmaking process remains warranted.

\section{Aim and Perspective of Economic Evaluations}

Economic evaluations have their roots in welfare economics, of which the central objective is to provide an ethical framework for making meaningful statements about whether specific (policy) changes improve social welfare. As Boadway and Bruce state: 'That is, the welfare economist wishes to determine the desirability of a particular policy-not in terms of his or her own values, but in terms of some explicitly stated ethical criteria' [8]. Welfare economics in that sense is inherently normative and one may argue that the art of applied health economic evaluations would benefit from more debate on the underlying ethical criteria. The current exchange of views hopefully contributes to that debate.

One of the important choices in any welfare economic evaluation is which elements of value are to be included in the analysis. In theory, all aspects of value that are relevant to the decision the analysis is trying to support should be included. This decision context, reflecting the goal of the decision maker, relevant constraints and decision-making process, differs between jurisdictions when it comes to health economic evaluations [9]. Some countries prescribe a societal perspective to be taken in economic evaluations. Such a perspective is in line with both welfare economic theory and the goal of optimizing social welfare. Then, all relevant costs and benefits, regardless of where, when, or on whom they fall, need to be included in the analysis [10]. Only by doing so, a full welfare economic evaluation can 
be performed and claims regarding the improvement of social welfare attempted to be made (difficult as they may be, given, for example, the 'difficulty' of specifying a full social welfare function).

Still, other jurisdictions take a narrower view of the decision-making aim, most notably maximizing or optimizing health from a given (fixed) budget [9]. Taking such a 'healthcare perspective' typically implies the inclusion of only those costs that fall under the healthcare (or sometimes social) budget and only health effects as benefits. This can be viewed as being in line with the budget responsibility of the informed decision maker and their assumed goal (health optimization $^{1}$ ). The perspective adopted and the assumed decision rule dictate the inclusion and exclusion of specific costs and benefits in the evaluation. Whereas direct (physician time, drug costs, etc.) and indirect (costs in gained life-years) medical costs are relevant in both perspectives, direct and indirect non-medical costs (e.g. travel costs and productivity costs, respectively) do not fall on the healthcare budget and would only be relevant when adopting a societal perspective.

Just to emphasize, adopting a societal perspective would, in principle, imply the inclusion of all influential costs and benefits. Taking a narrower perspective, such as the healthcare perspective, implies that aspects of real societal value are, by definition, left out of the analysis. Hence, decision makers are systematically left ignorant about the broader consequences of their decisions. Even if such consequences are seen as 'external effects' from the decision makers' perspective, it is clear that this risks reducing overall welfare and also (indirectly) health. For example, early discharge could (societally speaking, wrongfully) be pictured as cost effective because the costs (and effects) of informal care are ignored. Bed rest could be advised, over treatment, for some illnesses because lost workdays are not valued (or even considered). Even if one would accept that many earthly decision makers have a narrower scope than improving broad social welfare, leaving them ignorant about the nature, scope, and size of these external effects, as a rule, seems hard, if not impossible, to defend. This holds both in theory and in practice, especially in the context of allocation decisions.

The sometimes heard argument that equity requires such ignorance is used inconsistently, appears to ignore the fact that equity considerations should also be informed, that inclusion of broader elements of value can also improve

\footnotetext{
${ }^{1}$ I use the word 'optimization' rather than maximization to allow weighting of health gains, for instance to favor gains at the end of life, in young people or patients in poor health states. Such weights can be seen as reflecting differential social values of health gains. I emphasize that analogous to difficulties in specifying a social welfare function, it may be difficult to specify a full 'social health value function'.
}

equity, and that, ideally, the decision maker should (be able to) weight these arguments, informedly! This will be addressed more elaborately below.

\subsection{Spillover Effects}

In terms of relevant outcomes, effects, or benefits, the traditional focus has been on the inclusion of health effects in patients. These are obviously important outcomes in any analysis. Still, the scope of included effects appears to be broadening in recent years in at least two ways. First, it is becoming clear that health effects may not be the only relevant outcomes produced by the health and social care sector. For instance, in elderly care or palliative care, value can be created that may not be adequately reflected in QALY gains that only reflect health-related utility. New and broader outcome measures, including the ICECAP [11] and ASCOT [12] instruments, were developed in an attempt to fill this gap. Note that this broadens the evaluative scope of evaluations as well as their use in broader contexts, which may also have consequences for the perspective taken because the combination of broader outcome measures with 'only' healthcare costs may render difficult to interpret (if not meaningless) incremental cost-effective ratios. Second, relevant effects do not only occur in patients but also in others. Such effects are typically called spillover effects [1], which can have different characteristics and causes. Here, it is good to distinguish between family effects and informal caregiver effects, as well as between health and wellbeing effects [4].

Family effects occur in family members of the patient as a consequence of the health state of a loved one ('caring about' a patient) $[2,3]$. Examples include the influence of a serious illness of a child on the health and wellbeing of his/her parents, or the impact of mental health problems on the health and wellbeing of a partner. The impact of these effects can be large [6, 7]. Informal caregiver effects occur in caregivers due to performing physically or emotionally demanding care tasks, often over longer periods of time ('caring for' a patient). Numerous studies describe the effects of caregiving on the health $[13,14]$ and wellbeing $[2,15]$ of informal caregivers, highlighting the substantial impact of these effects. Given that informal caregivers are often family members of the patient, both types of effects are likely to occur in caregivers [4], and when investigating health effects in family members $[16,17]$, who may also be caregivers, both effects are likely to be present $[2,3]$.

Spillover effects are relevant for health economic evaluations adopting either a societal or healthcare perspective. From a healthcare perspective, the health effects in family members and caregivers are directly relevant for the decision rule that underlies the analysis: optimizing health from a fixed budget. Leaving out the health effects in significant others is inconsistent with this goal and risks decisions that 
reduce health. Hence, it is unsurprising that in the 2013 guidance of the National Institute for Health and Care Excellence (NICE) it was already mentioned that "... all direct health effects, whether for patients or, when relevant, carers" [18] should be included in the analysis. At that time, the focus, also in the literature, was mainly on health effects in informal caregivers; however, these effects also occur in the broader family network $[6,7]$. Ignoring these health effects risks missing the goal of health maximization or optimization that these decision-making bodies apparently aim for.

From a societal perspective, health effects are also relevant. In fact, the first US Panel on Cost-Effectiveness in Health and Medicine [10], recommending the adoption of a societal perspective, encouraged researchers to think broadly about the inclusion of health effects in significant others. Their inclusion in the analysis can be fairly straightforward [19], although more research into which health-related quality-of-life instruments are best suited to capture these effects remains important [20]. Broader wellbeing effects, affecting overall utility or welfare, not necessarily (only) health-related utility, are also relevant [3]. Their inclusion in economic evaluations, also for patients, is still less common and may be less straightforward, although is gaining attention [19]. Systematically ignoring elements of value that are relevant for and potentially influential in the decision cannot be justified in this context.

From a theoretical perspective, the inclusion of spillover effects is necessary in order to be able to make decisions in line with the specified goal. Practically, depending on the (relative) size and context of spillover effects, they may well make a difference for the final decision $[6,21]$. Hence, for reasons of efficiency, following the decision rules underlying economic evaluations from a healthcare or societal perspective, inclusion is warranted.

\subsection{Equity}

Systematically ignoring spillover effects cannot therefore be justified on the grounds that they do not make a difference or are irrelevant. Can they be ignored then because they do make a difference and thus have distributional consequences? In other words, would it be inequitable to include these effects in economic evaluations? Let me make two observations on that issue. First, inclusion of any cost or outcome variable can have distributional consequences. Take hospital costs. If elderly normally need to stay in hospital longer than younger people, for certain treatments, inclusion of hospital costs in the analysis may have distributional consequences. Would the right answer be to ignore these costs altogether then? Of course not. The decision maker needs to be informed about these aspects, including the distributional consequences in order to be able to make an informed decision in line with the specified goals, also trading off efficiency and equity goals where necessary. The argument to ignore specific cost aspects for reasons of equity seems to be used rather selectively. ${ }^{2}$ Fairness is not helped by ignorance, but by a full, adequate, and deliberative weighting of the issues at hand.

How about spillover effects? Their inclusion can, of course, have distributional consequences. The scope and nature of the consequences also depends on the type of decision normally taken. (Sometimes rather unlikely examples are used to argue against inclusion. For instance, only treating people from large families within one disease area seems an unlikely outcome of the decision-making process in many jurisdictions. More emphasis on treatments of those diseases with larger spillover effects compared with other diseases seems more realistic.) A crucial question here is whether the exclusion of spillover effects will lead to fairer decisions, as a rule. I would again argue this not to be the case. Take the health effects in informal caregivers. Ignoring these, while including the benefits of this care in terms of QALY gains of patients, is not only inconsistent but, in my opinion, excluding the adverse health effects in these valuable carers is also inequitable. These people sacrifice time, and often their wellbeing and health, to help patients. They deserve our attention in the decision-making process. Otherwise decisions (such as early discharge from hospitals) could lead to increased adverse health effects in caregivers, without consideration. It is hard to maintain that this is fair.

The same holds for the health (and wellbeing) impacts of health problems on family members, which can differ per type of disease. Why would it be fairer to not consider that in some diseases (e.g. mental health problems) spillover effects on family members, representing real health and wellbeing losses, are more prominent than in others? While fairness may involve weighting of these effects in a deliberative process, a case can certainly be made as to why fairness would benefit from their inclusion and consideration.

\footnotetext{
${ }^{2}$ Recently, it was, for instance, used to argue against the inclusion of medical costs in gained life-years [22], apparently largely motivated by the outcomes of inclusion (in one particular case). But again, ignoring these costs is inconsistent with the aims and methods of economic evaluations as it ignores real (health) opportunity costs of these expenditures [23]. It thus makes life-prolonging interventions seem more cost effective than they actually are and relatively more cost effective than quality-of-life-improving interventions. Is that (necessarily) fair, also for those people who will receive less care as a consequence? I would argue that ignoring them is not the appropriate answer to the problem, not consistent with the overall decision goals and methods, nor fairer as a rule! Deliberative consideration of these costs and their consequences is warranted.
} 


\subsection{Displacement?}

If the introduction of a new technology is believed to lead to displacement of existing care (or, more generally speaking, to health opportunity costs), this displacement will not only involve patient health and medical costs but also spillover effects and broader costs. It has been clearly illustrated how spillover effects can and should be included in such a context [7]. In short, both the gained and lost spillover effects need to be considered, leading to a consideration of "net spillover effects'. This is similar to the inclusion of other costs and effects when displacement is relevant.

One counterargument might be that we typically do not know what gets displaced. This is true, but, again, surely not unique for spillover effects. In the absence of guidance on disinvestments, what gets displaced is unknown and may also produce more patient health than what is allowed in the system [24]. This fact has not prevented us from making decisions, based on the available (limited) information on average opportunity costs, or from including patient health. So why would it justify ignoring spillover effects? One could argue that we know less about average spillover effects than about average marginal cost effectiveness of displacement. Well, if that is true, it is only more recently the case and would, moreover, be an excellent argument to include these effects. Inclusion will swiftly increase our knowledge about (average) spillover effects in different contexts. Broader investigations of spillover effects can complement this strategy. The more we learn about spillover effects, including how to best measure, value, and include them [17, 19], the better we will be in estimating (average) displaced spillover effects and the more weight (net) spillover effects can receive in the decision-making process. Again, ignoring the spillover effects does not improve our knowledge nor does this improve the trust we should place on decisions. Using the available knowledge may be considered superior over assuming net spillover effects to be zero as a rule, for efficiency and equity reasons. Inclusion in that sense at least allows us to be 'vaguely right' rather than being 'precisely wrong' with exclusion, and to become increasingly precisely right. More research into the measurement, valuation, and inclusion of spillover effects can facilitate this [25].

\subsection{Concluding}

Spillover effects are real and potentially large health and wellbeing effects in caregivers and family members. If our aim is to improve health or welfare, ignoring such effects in economic evaluations risks making decisions that do not improve health or welfare. Moreover, ignoring these effects does not, as a rule, result in fairer decisions. Full consideration of relevant aspects is necessary for reasons of efficiency and equity, as well as their trade-off. The inclusion of spillover effects in economic evaluations and their deliberative consideration in the decision-making process should therefore be the norm.

\section{Rebuttal to McCabe}

While I welcome Chris McCabe's commentary [26], it deserve a few words of caution of its own, especially because his suggestions may well harm rather than improve health, welfare, and equity. Here, I will briefly clarify some incorrect and inconsistent claims, 'criss-crossing' through his commentary.

\subsection{No Regard for Opportunity Costs?}

McCabe's commentary suggests that broadening the scope of evaluations would ignore opportunity costs and displacement. This is incorrect. On the contrary, in a societal perspective everyone affected by a decision should be considered. Taking a broader perspective is fully compatible with accounting for fixed budgets and displacement when relevant [9]. A framework for the inclusion of spillover effects in such a context has already been proposed [7]. Meanwhile, evidence on spillover effects is increasing, as this special issue of PharmacoEconomics testifies, making it increasingly possible to have an idea about 'net spillover effects' whenever deemed relevant. Moreover, it seems obvious that McCabe's suggestion of excluding spillover effects from economic evaluations and decision making is not an answer to the issue of opportunity costs and risks health lowering, inefficient and unfair decisions.

\subsection{Uncertainty}

Including spillover effects (like any other component in an economic evaluation) may increase uncertainty, but uncertain relevant estimates are certainly better than certain irrelevant estimates of zero! Decision makers can (be trusted to) use the presented evidence prudently, also considering the uncertainty. Moreover, the uncertainty McCabe accepts regarding whether and which patient health is displaced (i.e. we normally do not know what gets displaced: “... decision makers assume that it is the least valuable health care activity that is displaced...") [26], seemingly is unacceptable when it comes to caregivers' health, the result being that their health gets ignored and potentially harmed. This also already signals arbitrary choices in his approach.

\subsection{Arbitrary and Inconsistent}

McCabe indeed draws arbitrary lines around what he wants to consider in a normative analysis. Take, for example, his 
discussion of the inclusion of medical costs occurring during life-years gained. Exclusion is inconsistent with the aim of health maximization he himself adopts, as it ignores real health opportunity costs [12]. Important distributive issues should of course be addressed, but ignoring real costs and effects risks both inefficiency and inequity.

His most crucial inconsistency is related to the statement regarding the purpose, as McCabe sees it, of economic evaluations: "The focus is on the health produced by the technologies, not any characteristic of the individuals who receive or lose health. ... The key point is that decision makers are indifferent to who receives and loses health; it is maximizing the health produced that matters" [26]. If these two statements are true, then why the distinction between health produced (and lost) in patients versus health produced (and lost) in others like caregivers? These health effects are equally real and important under this rule and our effort should be on collecting the relevant evidence to make the best decision possible given the agreed aims (which may include equity considerations).

The inclusion of spillover effects is therefore fully consistent with these broad aims. Ignoring (health) effects is not! McCabe writes that the "appropriate scope for costs and benefits should rely on the scope of costs and benefits identified as relevant by the decision maker for whom the analysis is undertaken". NICE, perhaps most prominent in functioning under the assumption of a fixed budget, was, already in 2013, suggesting to include " $\ldots$ all direct health effects, whether for patients or, when relevant, carers" [18].

Moreover, while I fully acknowledge the ultimate responsibility of decision makers in setting the scope and process for their decisions, I do think they should (and often want to) be informed by research on how to optimally do so. Take McCabe's example of presenting ministers deciding on additional taxation for funding new healthcare interventions with a full account of costs and benefits. To subsequently fail to do the same when spending the money raised on the basis of this full account, by taking a narrower scope by a subsequent decision maker, clearly risks inconsistent and welfarelowering decisions. Understanding this may help to improve decision-making processes, which we cannot dictate (fortunately) but can and should inform.

\subsection{Procedural Justice}

McCabe emphasizes the need for (procedural) justice, and I agree. He argues (my phrasing) that because we do not know what gets displaced, we should not include spillover effects since the (caregivers of the) people whose care is displaced do not have a voice in the process. I note two things in response. First, since we do not know what gets displaced, patients harmed by displaced or forgone activities have no direct voice in the procedure either. They are represented in the estimates of opportunity costs. The same can be done for spillover effects [7]. Differences in certainty about the estimates can be reflected in decision-making weights and procedures. Second, judging whether something leads to better or worse procedures and decisions depends on the comparator. McCabe's alternative is to ignore real spillover effects in decision making altogether. Suggesting this gives more voice to family members and caregivers or, as a rule, leads to better decisions, seems heroic. Rather, McCabe effectively places zero weight on changes in health in others in the decision-making framework by excluding them (both for new and displaced care!). I fail to see how this would be more procedurally fair or consistent with his own aims.

\subsection{Concluding}

McCabe's words of caution are welcomed, and indeed any consideration of spillover effects should be done carefully, fairly, and prudently. Nothing in his argumentation provides a convincing argument to exclude these effects from economic evaluations. In fact, exclusion is even inconsistent with the purpose he himself describes for economic evaluation. Caution is therefore especially required to avoid ignoring spillover effects. So, let's agree to proceed with caution by (fairly, prudently, and explicitly) including (net) spillover effects in all future health economic evaluations!

\section{Compliance with Ethical Standards}

Funding No funding was received for this commentary and rebuttal.

Conflict of interest Werner Brouwer has no conflicts of interest other than a long-standing history of advocating the societal perspective in economic evaluations.

\section{References}

1. Basu A, Meltzer D. Implications of spillover effects within the family for medical cost-effectiveness analysis. J Health Econ. 2005;24:751-73.

2. Bobinac A, Van Exel J, Rutten F, Brouwer W. Caring for and caring about: disentangling the caregiving effect and the family effect. J Health Econ. 2010;29(4):549-56.

3. Bobinac A, Van Exel J, Rutten F, Brouwer W. Health effects in significant others: separating family and caregiving effects. Med Decis Mak. 2011;31(2):292-8.

4. Brouwer WBF, Tilford M, van Exel NJA. Incorporating caregiver and family effects in economic evaluations of child health. In: Ungar W, editor. Economic evaluation in child health. Oxford: Oxford Press; 2009.

5. Hoefman RJ, van Exel NJA, Brouwer WBF. How to include informal care in economic evaluations. Pharmacoeconomics. 2013;31(12):1105-19.

6. Al-Janabi H, van Exel NJA, Brouwer WBF, Trotter C, Glennie L, Hannigan L, et al. QALY losses in patients' family networks: 
a study of the wider health effects of meningitis. Health Econ. 2016;25(12):1529-44.

7. Al Janabi H, van Exel NJA, Brouwer WBF, Coast J. A framework for including health spillovers in economic evaluation. Med Decis Mak. 2016;36(2):176-86.

8. Boadway R, Bruce N. Welfare economics. Oxford: Basil Blackwell; 1984

9. Claxton K, Paulden M, Gravelle H, Brouwer W, Culyer A. Discounting and decision-making in the economic evaluation of healthcare technologies. Health Econ. 2011;20:2-15.

10. Gold M, Siegel J, Russell L, Weinstein M. Cost-effectiveness in health and medicine. New York: Oxford University Press; 1996.

11. Grewal I, Lewis J, Flynn T, Brown J, Bond J, Coast J. Developing attributes for a generic quality of life measure for older people: preferences or capabilities? Soc Sci Med. 2006;62:1891-901.

12. Netten A, Burge P, Malley J, Potoglou D, Towers A, Brazier J, et al. Outcomes of social care for adults: developing a preferenceweighted measure. Health Technol Assess. 2012;16(16):1-165.

13. Do YK, Norton EC, Steams SC, Van Houtven CH. Informal care and caregiver's health. Health Econ. 2015;24(2):224-37.

14. Schulz R, Beach SR. Caregiving as a risk factor for mortality: the Caregiver Health Effects Study. JAMA. 1999;282(23):2215-9.

15. Hoefman RJ, van Exel NJA, Brouwer WBF. Measuring the experienced impact of informal care on carers: a construct validation study of the CarerQol instrument in a large sample of caregivers. Health Qual Life Outcomes. 2013;11:173.

16. Wittenberg E, Ritter GA, Prosser LA. Evidence of spillover of illness among household members: EQ-5D scores from a US sample. Med Decis Mak. 2013;33(2):235-43.

17. Wittenberg E, Prosser LA. Disutility of illness for caregivers and families: a systematic review of the literature. Pharmacoeconomics. 2013;31(6):489-500.
18. NICE. Guide to the methods of technology appraisal. London: NICE; 2013

19. Hoefman RJ, van Exel NJA, Brouwer WBF. How to include informal care in economic evaluations. Pharmacoeconomics. 2013;31(12):1105-19.

20. Payakachat N, Tilford JM, Brouwer WBF, van Exel NJA, Grosse SD. Measuring health and well-being effects in family caregivers of children with craniofacial malformations. Qual Life Res. 2011;20(9):1487-95.

21. Krol M, Papenburg J, van Exel NJA. Does including informal care in economic evaluations matter? A systematic review of inclusion and impact of informal care in cost-effectiveness studies. Pharmacoeconomics. 2015;33(2):123-35.

22. Grima DT, Bernard LM, Dunn ES, McFarlane PA, Mendelssohn DC. Cost-effectiveness analysis of therapies for chronic kidney disease patients on dialysis: a case for excluding dialysis costs. Pharmacoeconomics. 2012;30(11):981-9.

23. van Baal PHM, Meltzer D, Brouwer WBF. Pharmacoeconomic guidelines should prescribe inclusion of indirect medical costs! A response to Grima et al. Pharmacoeconomics. 2013;31(5):369-73.

24. Barrett A, Roques T, Small M, Smith RD. How much will Herceptin really cost? BMJ. 2006;333(7578):1118-20.

25. Tilford JM, Payakachat N. Progress in measuring family spillover effects for economic evaluations. Expert Rev Pharmacoecon Outcomes Res. 2015;15(2):195-8.

26. McCabe C. Expanding the scope of costs and benefits for economic evaluations in health: some words of caution. Pharmacoeconomics. 2018. https://doi.org/10.1007/s40273-018-0712-8. 